\title{
Die Schnitzkunst im Leben der Afo von Nord-Nigeria
}

\author{
Elsy Leuzinger ${ }^{1}$
}

Die Afo bewohnen das Bergland von Nasarawa im sogenannten Middle Belt der Benue-Provinz. Ihr Gebiet grenzt im Süden an den Benue, den großen Nebenfluß des Niger, im Osten an die Lafia- und Keffi-Emirate, im Norden und Nordwesten an das Nasarawa-Emirat und im Westen an den unberührten Busch. Die Siedlungen sind nur auf schmalen Pfaden zu Fuß erreichbar.

Diese Afo bieten geradezu ein Musterbeispiel einer unverfälschten einfachen Kultur von Jägern-Ackerbauern, die ihre angestammte Lebensweise allen Einwirkungen der sie umgebenden höher zivilisierten Negerstämme zum Trotz in großartiger Geschlossenheit bewahrt haben. In stolzem Unabhängigkeitswillen stellen sie sich sowohl gegen Islam und Christentum wie gegen alle Einwirkungen der Zivilisation. Meine Partnerin und ich konnten uns deshalb glücklich schätzen, in diesem geschlossenen Stammesgebiet überhaupt Einlaß gefunden zu haben. Es gelang dies nur, weil die Frauen bei den Afo keinerlei politische und religiöse Funktionen haben und diesbezüglich als völlig ungefährlich gelten, und weil wir versicherten, als Lernende und nicht als Missionierende gekommen zu sein. Es mag sie auch etwas Neugier bestochen haben, weil die meisten Afo noch nie weißen Frauen begegnet waren.

Die Afo sind Semibantu mit äthiopidem Einschlag. Der etwa 10000 Seelen zählende Stamm wohnt in 20 Dörfern. Jedes dieser Dörfer setzt sich aus mehreren umfriedeten Familiengehöften zusammen, ist autonom und wird nach außen durch einen Häuptling vertreten. Innerhalb seiner Gemeinschaft hat dieser Häuptling die Macht jedoch mit dem Rat der Alten, dem Dorfältesten und seinem voraussichtlichen Nachfolger zu teilen. Die Organisation ist ausgesprochen vaterrechtlich, und die erste Häuptlingsfrau leitet die von den Räten erlassenen Gebote an die Frauen weiter. Die Angaben der Afo über ihre Vergangenheit sind unklar. Einerseits betrachten sie das Nasarawa-Bergland als ihre Heimat, die sie schon seit mehreren hundert Jahren innehaben. Anderseits erklären sie ausdrücklich, früher im Adamaua-Hochland südlich des Benue gelebt zu haben, noch im Stadium eines primitiven Jägerdaseins von dort ausgewandert und den Flüssen Taraba, Donga und Katsena entlang nordwärts gezogen zu sein. In Lafia hätten sie sich mit Bornu-Leuten der Tschadregion vermischt und hierauf im Nasarawa-Bergland niedergelassen. Die Zeit ist ihnen noch im Gedächtnis, als sie als Jäger in Felsennischen hausten und sich mit Steinen verteidigten, da sie immer wieder Kämpfe mit den Haussa auszufechten hatten. Vor etwas über 100 Jahren hatten sie begonnen, das Busshland am Fuß der Felsen zu roden und Felder anzulegen. Ihre Wohnstätte behielten sie jedoch noch im Gebirge, erbauten aber Häuser aus Stein. In jüngster Zeit endlich, als durch die "pax britannica» die Schutzlage im Gebirge nicht mehr nötig war, hatten sie auch ihre Wohnstätten an den Rand der Berge verlegt. Das Handwerk, so erzählen die Afo weiter, hätten sie erst vor 100 Jahren von den «Bornu-Leuten" erlernt. Interessanterweise fiel in diesem $\mathrm{Zu}$ sammenhang der Name Adâma, eines heiligen Mannes, der ihnen den Schmalbandwebstuhl gebracht haben soll. Adâma aber ist der Name eines berühmten Fulani-Heerführers, der in der ersten Hälfte des 19. Jahrhunderts in mohammedanischem Glaubenseifer die heidnischen Stämme der Benue-Region eroberte. Bei der großen Völkerverschiebung, die er auslöste, hatten sich viele der heidnischen Stämme ins Gebirgsland südlich des Benue geflüchtet. Der Staat, den Adâma gründete, wurde nach ihm Adamaua genannt. Auch die Afo sollen vor dieser Fulani-Bedrohung in die südliche Benue-Region ausgewichen, später aber wieder daraus vertrieben worden sein. Sprachliche und kulturelle Bande verknüpfen sie mit dort ansässigen Stämmen, besonders mit den Arago, Agato und Idoma.

Die Isolation des Busches, die sich die Afo wählten und ihr spezifischer Partikularismus hatten zur Folge, daß all ihre technischen Errungenschaften in den Anfängen steckenblieben. Es wird stetsfort auf der Bogengeige musiziert - Prototyp von Geige und Harfe - und überall stehen Fetische. Angesichts dieses interessanten, altertümlichen Kulturstandes lag es nahe, bei den Afo nach etwelchen Ursprüngen der Kunst zu forschen und sich die folgenden Fragen zu stellen:

1. Werden von Menschen dieser relativ niedrigen Kultur wirkliche Kunstwerke geschaffen?

2. Was wäre ihr Sinn?

3. Wie sind die Afo zur Form gelangt? Können wir bei Ihnen etwa noch das Werden der Form erkennen, eine primitive Kunst im Sinne von «ursprünglich» und «naiv»? 
Um diese Fragen zu beantworten, sei zuerst das Fundmaterial skizziert, dann die Funktion der religiösen Skulptur im Leben der Afo aufgezeigt und die Form zweier Schnitzwerke interpretiert. Zuletzt wird zu ermitteln versucht, woher die Impulse stammen, ob die Form eigenständig ist oder ob sie in einem größeren Kulturzusammenhang gesehen werden muß.

\section{Die künstlerische Produktion}

Zierfreudigkeit äußert sich bei den Afo in bescheidenem Maße, sowohl in der Tracht wie an den Hauswänden und Gerätschaften. Die Frau läßt ihren Körper nach der Phantasie des Operateurs mit dekorativen Narbenmustern übersäen (Abb. 1). Der Ornamentschatz reicht kaum über die einfachsten geometrischen Grundmuster hinaus: Rillen, Dreiecke, Rauten, Fischgräte oder Gitter sind die Regel; gerne wird Kerbschnitt angebracht, wie zum Beispiel bei den hölzernen Hockern (Abb. 2). Selten nur werden einfache Tierumrisse mit einbezogen (Abb. 12/13) oder die randständigen Bogen und Eckschleifen der Haussa in die Kalebassen und hölzernen Sandalen der Würdenträger eingeschnitten (Abb. 3). An Zaubergeräten bemerkt man öfters den Zierbesatz von roten Abrusbohnen und weißen Kaurischnecken, die mit Honig angeklebt werden.

Das Handwerk wird nach den Geschlechtern getrennt ausgeübt. Die Männer flechten, weben und schnitzen; sie erbauen die Häuser und stellen die Schmiede. Den Frauen obliegt das Spinnen des Baumwollgarns, das Färben der Stoffe mit Indigo und das Töpfern ohne Töpferscheibe und Brennofen. Sie formen die großen Tonkrüge aus freier Hand und rollen ein Schnurstück auf dem noch weichen Ton der Gefäßwand $a b$, um eine regelmäßige Musterung zu erzielen: Schnurkeramik noch heute!

Besondere Aufmerksamkeit verdienen die im Cireperdue-Verfahren gegossenen, reich verzierten Messingpfeifen mit lederumwundenem Rohr. Sie gelten als Würdezeichen der Ratsmitglieder. Die Afo wollen solche Pfeifen und andere Messingobjekte früher selbst fabriziert haben. Heute ist diese Technik bei ihnen jedoch völlig verschwunden. Der Messingguß wird bei mehreren Stämmen der Benue-Region ausgeübt, in ähnlichen Formen bei den Idoma, Igala und bei den als Gelbgußkünstler besonders berühmten Tiv. Ein bedeutendes Messingguß-Zentrum war Lokoja.

Freies Zeichnen und Malen kennen die Afo nicht. Und unter den vielen vom Medizinmann verfertigten Fetischen ragt nur weniges über eine unbekümmerte naive Primärkunst hinaus. So ließe es sich bei all dem, was sich dem Betrachter in einem Afo-

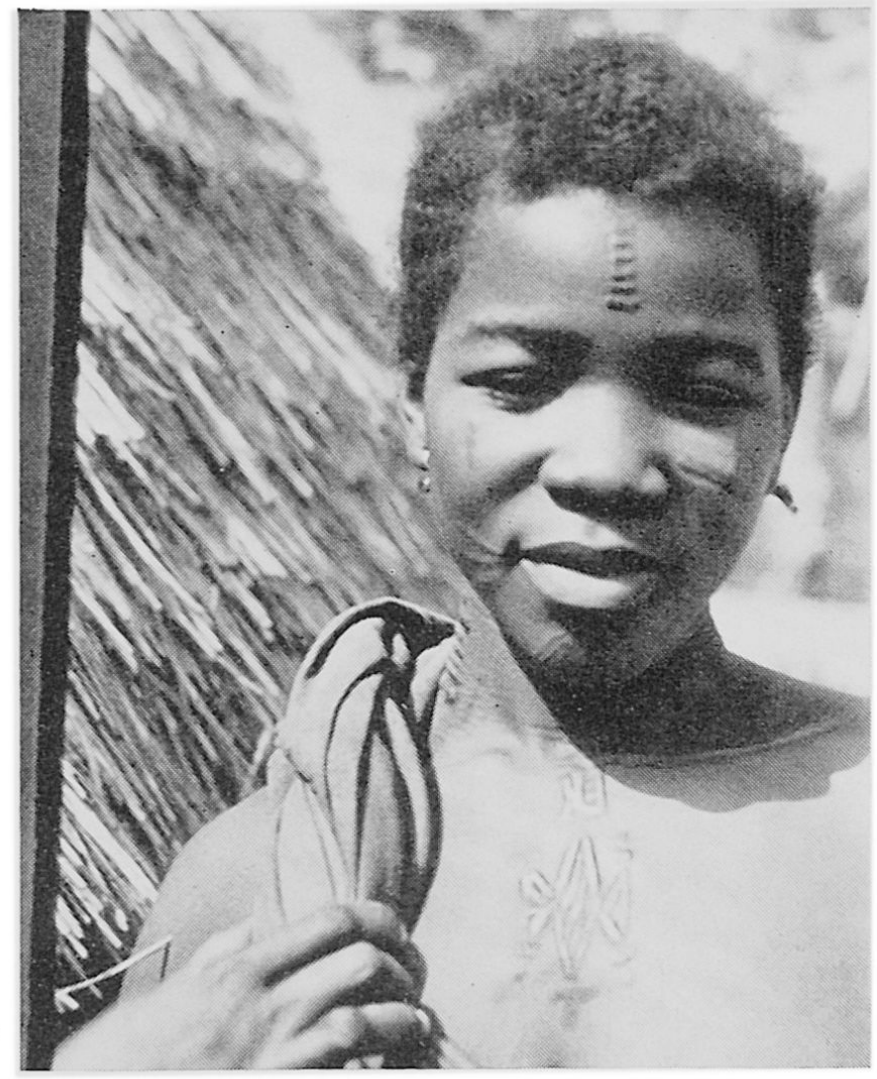

Abb. 1 Afo-Frau mit Narbenmustern

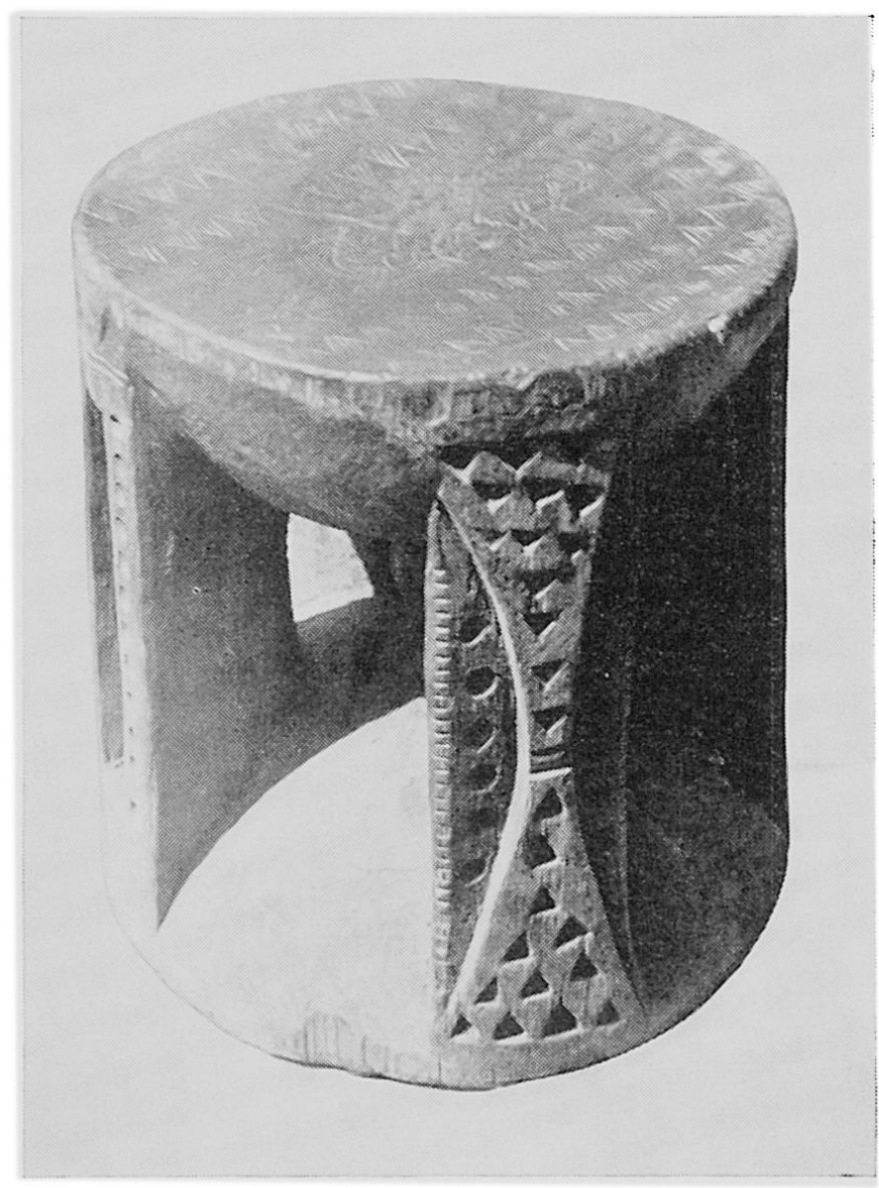

Abb. 2 Häuptlingssitz mit Kerbschnitt; Holz 


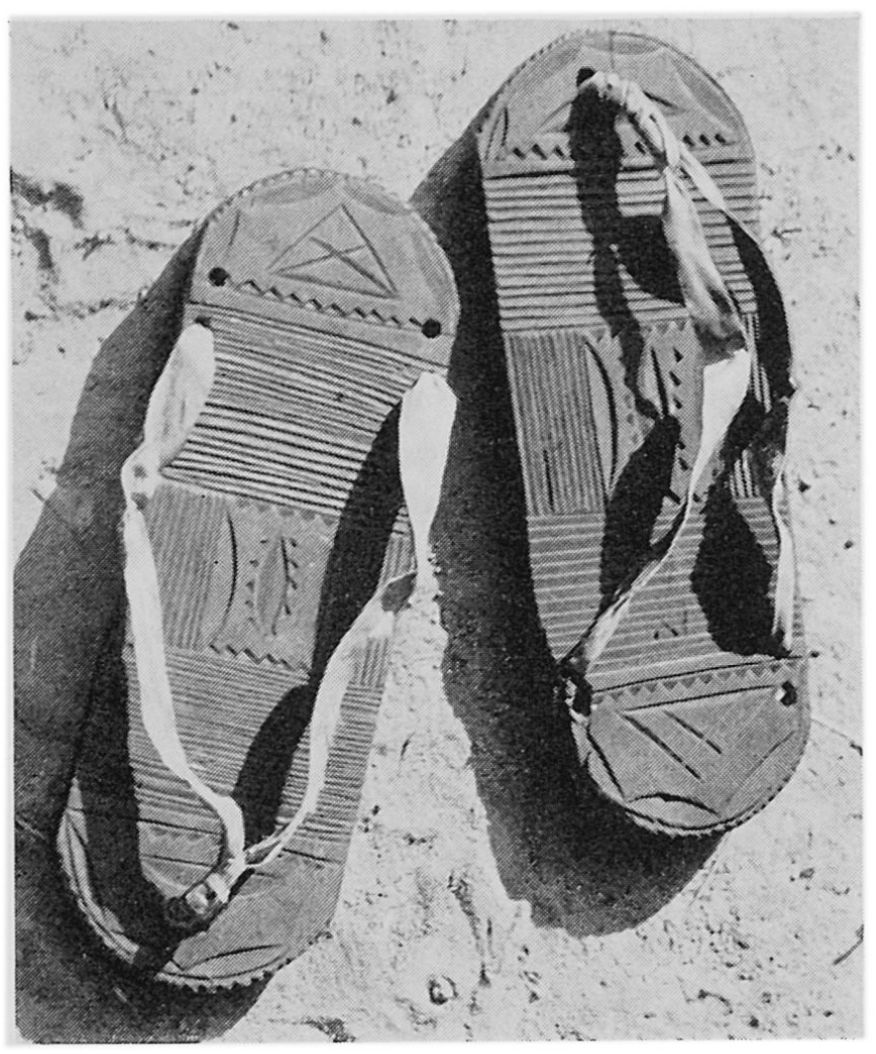

Abb. 3 Sandalen aus Holz, mit Lederriemen

Negerdorf auf den ersten Anhieb darbietet, nicht rechtfertigen, von einer wirklichen Kunst zu sprechen. Allein, dank unserem langen Aufenthalt und dem allmählich gewonnenen Vertrauen war es uns vergönnt, Einblick in das verborgenste Schaffen der Männer zu gewinnen und zu anderen Schlüssen zu gelangen.

Es geschah im Dunkel der Nacht, daß uns die AfoMänner ihre mächtigen Masken, Tanzaufsätze und Statuen zeigten. Sie brachten sie in Bündel verpackt in unsere Hütte und enthüllten sie in aller Heimlichkeit, damit ihre Frauen sie nicht sehen konnten. Denn sie lassen diese im Glauben, daß sie beim Anblick der kraftgeladenen heiligen Bildwerke sterben würden. Diese Schnitzereien nun ragen weit über das Gewöhnliche hinaus. Auch ihrem Sinn kamen wir allmählich auf die Spur: sie stehen alle im Dienst der Religion.

\section{Funktion der religiösen Plastik in der Gemeinschaft}

Für die Afo ist das gesamte Universum von der mythischen Lebenskraft durchdrungen, die von "Ukpu», dem entfernt im Himmel thronenden Schöpfergott und "Meister aller Seelen», ausgestrahlt wird. Ukpu manifestiert sich am stärksten durch seine "Söhne», große Geister, die sich den Menschen auf vielfache Weise offenbaren: durch "Abo", den mächtigen im Busch wohnenden Zauberer, der bei wichtigen Anlässen im Maskenkleid erscheint, "Ogäläbä», der den Menschen nie sichtbar wird, seine unnatürliche Stimme aber aus dem Busch ertönen läßt, und "Ängä», die heilige Buschkuh, Sohn des Abo und gütige Schutzmacht des Dorfes. Diese Buschkuh wurde uns als braunes Wildrind mit spitzen Hörnern beschrieben, dem möglicherweise die Vorstellung des kleinen, starken Waldbüffels zugrunde liegt. Im Vollgefühl der Verbundenheit mit den großen und kleinen Göttern, den Geistern und all den Totenseelen unterstellen sich die Afo ihrem Gebot. Sie erbitten ihren Schutz gegen Dämonen und vertrauen dem Medizinmann, $\mathrm{da} ß$ er sie mit Hilfe der Buschkuh meistert. Jedes Dorf hat seinen Medizinmann, der die traditionellen bewährten Methoden kennt, um den Geistern wirkungsvoll begegnen zu können, sie zu ehren und zu lenken. Als wichtiges Hilfsmittel dient ihm dabei die Skulptur; sie ist Katalysator der Lebenskraft.

Jedes Dorf besitzt eine ungefüge Tierplastik aus Lehm als Abbild der heiligen Buschkuh (Abb. 4). Sie steht in einem eigens für sie erbauten Rundhaus mit Doppelkonus und vorgelagertem Steinkreis (Abb. 5). Der Medizinmann opfert vor dem Lehmbild das Blut von Hörnertieren; denn die Buschkuh spendet Fruchtbarkeit für Frau und Feld. Den Frauen ist das Betreten des Heiligtums bei Todesstrafe verboten. Den Männern gelingt es vorzüglich, durch die Geheimnistuerei um diesen hohen Geist den Respekt zu wahren und das andere Geschlecht so einzuschüchtern, daß es nicht rebelliert. Die Buschkuh wird auch durch kühn konzipierte Masken dargestellt (Abb. 6, 7). Besuchen die Würdenträger eines Dorfes in offizieller Mission ein anderes Dorf, so geht ihnen ein Maskenträger voraus, um die Gäste als große, gute Macht zu kennzeichnen.

Zur Darstellung der Totengeister verwenden die Afo weiße und schwarze Masken mit menschlichen Gesichtszügen (Abb. 8, 9), manchmal mit Bart als Würdezeichen. Sie erscheinen beim Begräbnis, um den Trauernden aus dem Jenseits Trost und Rat zu bringen, und sie haben ebenfalls die Kraft, Dämonen zu bannen. Auch beim alljährlichen Totenfest läßt man sie auftreten, damit sie Kinder senden sowie nach der Ernte, um Dank und Opfer entgegenzunehmen.

Die Tänzer sind durch ein Netzkostüm verhüllt oder in die Festtracht der Haussa gekleidet. Ein weiblicher Totengeist im Netzkostüm begleitet sie auf Stelzen, um unheimlich zu wirken. Das Ziel all dieser Mimen ist es ja, möglichst übermenschlich auszusehen. Geisterhaft soll auch ihre Stimme sein, die durch ein Geheiminstrument im Munde - ein mit einer Spinnmembran verschlossenes Kürbisdöschen - erzeugt wird. Im Maskenkleide ist der Tänzer nicht mehr er selbst; er fühlt sich von der göttlichen Macht durchdrungen und gerät durch 


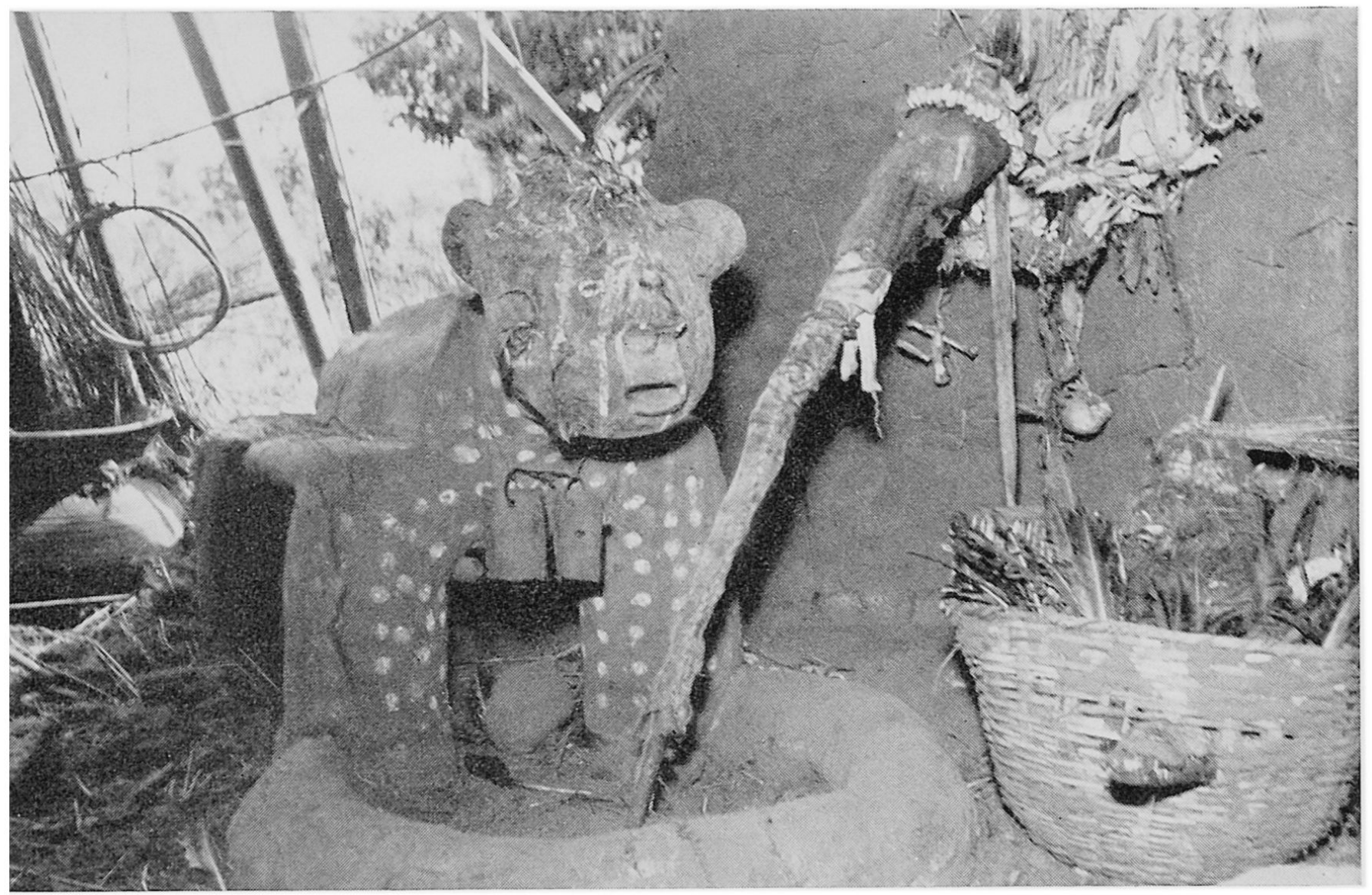

Abb. 4 Lehmplastik der heiligen Buschkuh

die straffen Rhythmen der Trommeln, Rasseln und Eisengongs rasch in Ekstase.

Eine ähnliche Funktion wie die Masken haben auch die hölzernen, schwarzen Tanzaufsätze der Afo (Abb. 11); auch sie werden bei Ernte und Tod getragen. Sie stellen aber weder Gottheiten noch Ahnen dar, sondern sind nur mit Symbolen der Kraft ausgestattet, so etwa mit Hahnenkamm, Rhinozerosohren, Tierhörnern oder dem Bild des unheimlichen Chamäleons. Abrusbohnen geben den roten Farbakzent. Der Hiahn gilt als Bote des höchsten Gottes und ermahnt diesen an sein Versprechen, stets für reichliche Nahrung besorgt zu sein. Das Chamäleon gilt auch bei den Afo ob seiner langsamen Bewegungen und des Farbwechsels willen als verhext. Je nach seiner momentanen Farbe bringt es Tod, langes Leben, Reichtum oder Kindersegen. Sein Zauber befähigt den Häuptling, nach Chamäleonsart ruhig zu bleiben und seine Farbe nicht in zorniges Rot hinüberzuwechseln. In diesem Zusammenhang sei erwähnt, daß die im Kontakt mit den Afo stehenden Fulani das Chamäleon als Stammestier verehren. Die Tanzaufsätze vereinigen die genannten Formelemente oft in raffiniert dekorativer Weise. Es sind abstrakte Kompositionen, die eine entfernte, wohl nur zufällige Ähnlichkeit mit dem Suguni-Typus der Tjiwara haben, den bekannten Antilopen-Tanzaufsätzen der Bambara im Westsudan. Eine andere Beziehung ist jedoch aufschlußreich. Das seitlich aus dem "Rhinozerosohr», dem Rund, aufsteigende Hörnerpaar ist ein ausgefallenes Formelement, das auch bei den "Ikenga» der Igala, Idoma, Ibo und anderer südnigerianischer Stämme zu beobachten ist². Solche Ikenga gelten als Sitz der Lebenskraft, sind also Kraftsymbole wie die schwarzen Tanzaufsätze der Afo.

Eine wichtige Funktion im Kult der Afo hat auch die große Mutter-Kind-Statue, die jeweils Gemeingut des Dorfes ist (Abb. 12). In ihr wohnt eine für die Fruchtbarkeit besonders wirksame Gottheit. Während des Jahres liegt die Figur in Tüchern verpackt verborgen in einem Tempelchen und wird nur vom Priester betreut. Einmal im Jahr holt er sie heraus, schmückt sie, übergießt sie mit Palmöl und Opferblut und stellt sie in den Steinkreis des Dorfplatzes. Erst jetzt werden die Frauen herbeigerufen, damit sie Geschenke bringen und in angemessener Distanz um die Statue wandeln. Vorher dürfen die Frauen sie nicht sehen, denn sie sollen nicht merken, daß das Götterbild gar nicht lebt, daß es nicht selbst gehen und sprechen kann. Die Männer befinden sich im innern Kreis, singen, trommeln und beschwören die der Figur innewohnende Kraft, sie möge ihren Frauen viele Kinder schenken. 


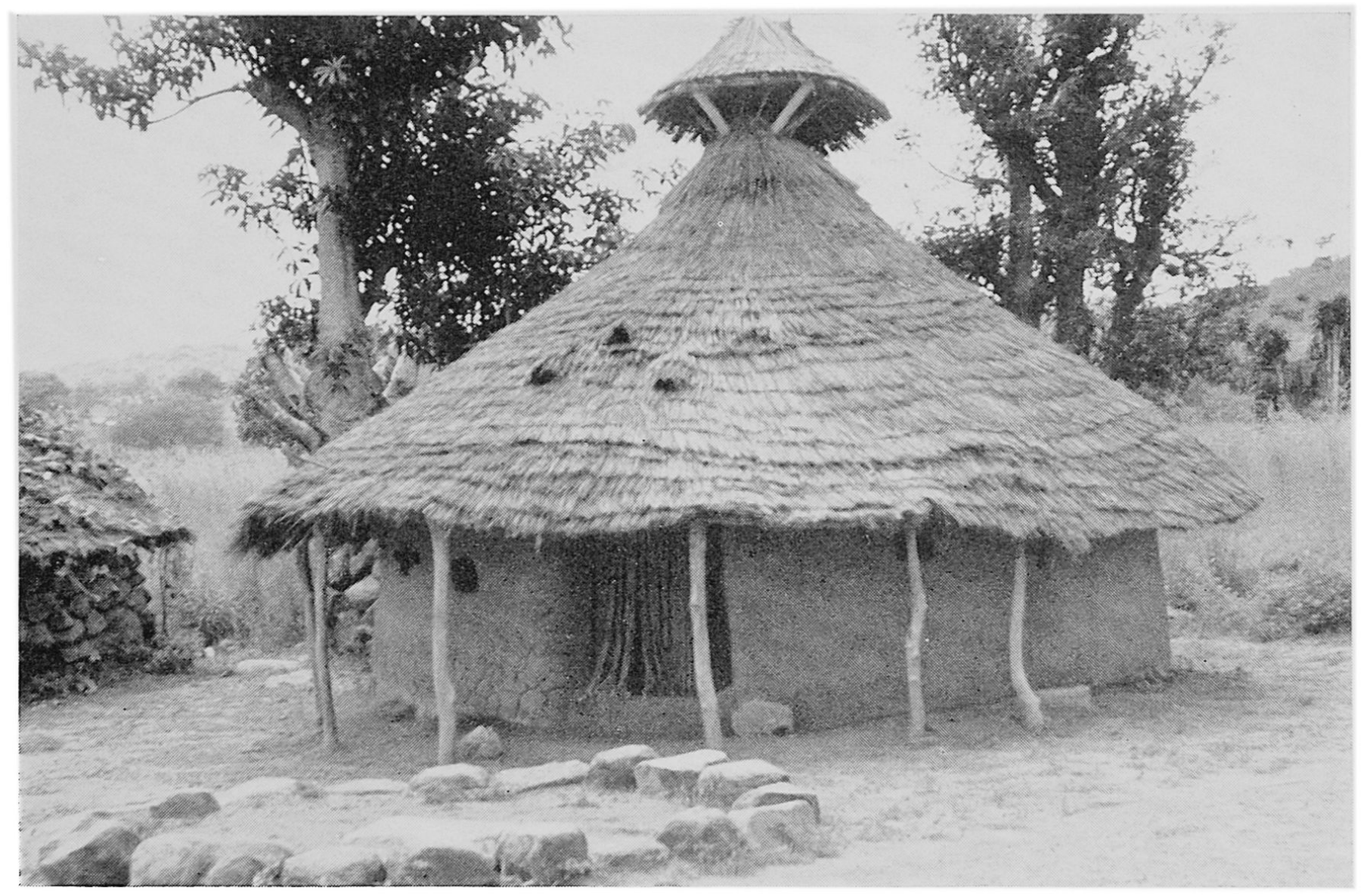

Abb. 5 Heiligtum der Buschkuh mit doppelkonischem Dach und Steinkreis

Deshalb die Darstellung einer schwangeren Frau. Die Afo sagen, die Fruchtbarkeit liege in den Brüsten, solange sie Milch spenden, die geistigen Kräfte und die Intelligenz wären im großen Kopf und ein großer Nabel bedeute viele kräftige Kinder, denn da gehe die Seele aus und ein. Eine der schönsten Statuen, die vermutlich noch heute in ihrem Wirkungskreis steht, wurde uns in aller Heimlichkeit gezeigt.

Es wurde uns leider nicht erlaubt, einem der Künstler bei der Herstellung einer solchen Statue zusehen zu dürfen. Das Schnitzen ist eine derart sakrosankte Angelegenheit, daß kein Uneingeweihter zusehen darf. Der Afo glaubt, daß er der Baumseele durch das Fällen und Schlagen des Stammes Schmerzen bereitet, und er begleitet deshalb die Arbeit unablässig mit Zeremonien und Opfern, um diesen Geist zu beschwichtigen. Der geringste Fehltritt würde des Schnitzers Tod oder eine gefährliche Krankheit zur Folge haben. Die große Gefahr, der sich der Künstler während der Arbeit ausgesetzt glaubt, soll mit ein Grund sein, weshalb sich heute kaum mehr einer zu schnitzen getraut und die Statuen lieber durch simple Graspuppen ersetzt. Die hohe Gefühlsspannung, die der Künstler während der Arbeit im Bannbereich des Göttlichen durchsteht, ist indessen eines der Geheimnisse, weshalb die religiöse Skulptur in ihren Sternstun- den zu hoher Ausdruckskraft gelangt. Das fertige Werk wird jeweilen noch im Feuer geschwärzt und mit Honig eingerieben, um das Springen des Holzes zu verhindern.

Früher gingen begabte Knaben zu einem berühmten Schnitzer in die Lehre, um durch Nachahmung des Vorbildes zu lernen und in die komplizierten Regeln und Riten eingeweiht $\mathrm{zu}$ werden, ging es doch um lebenswichtige Kunst für Schutz und Fruchtbarkeit des Stammes. Der Schüler hatte als Entgelt eine Zeitlang auf dem Felde des Lehrers zu arbeiten, ihm Ziegen und Hühner zu bringen und auch später, bei eigenem Verdienst, ihm immer wieder Teilerträgnisse abzugeben.

\section{Interpretation der Form zweier Skulpturen}

Um die künstlerische Qualität der Afo-Kunst darzulegen, ist gewiß diejenige Fruchtbarkeitsstatue (Abb. 12, 13) besonders geeignet, welche die Afo über alles schätzten und um keinen Preis veräuBert hätten; denn keiner ihrer Schnitzer, so sagten sie, könnte je wieder etwas Ähnliches schaffen. Es ist ein Bildwerk, das allgemeine Wesenszüge einer guten Negerplastik in sich vereinigt: die Geschlossenheit der Form - es wirkt die Grundform des 


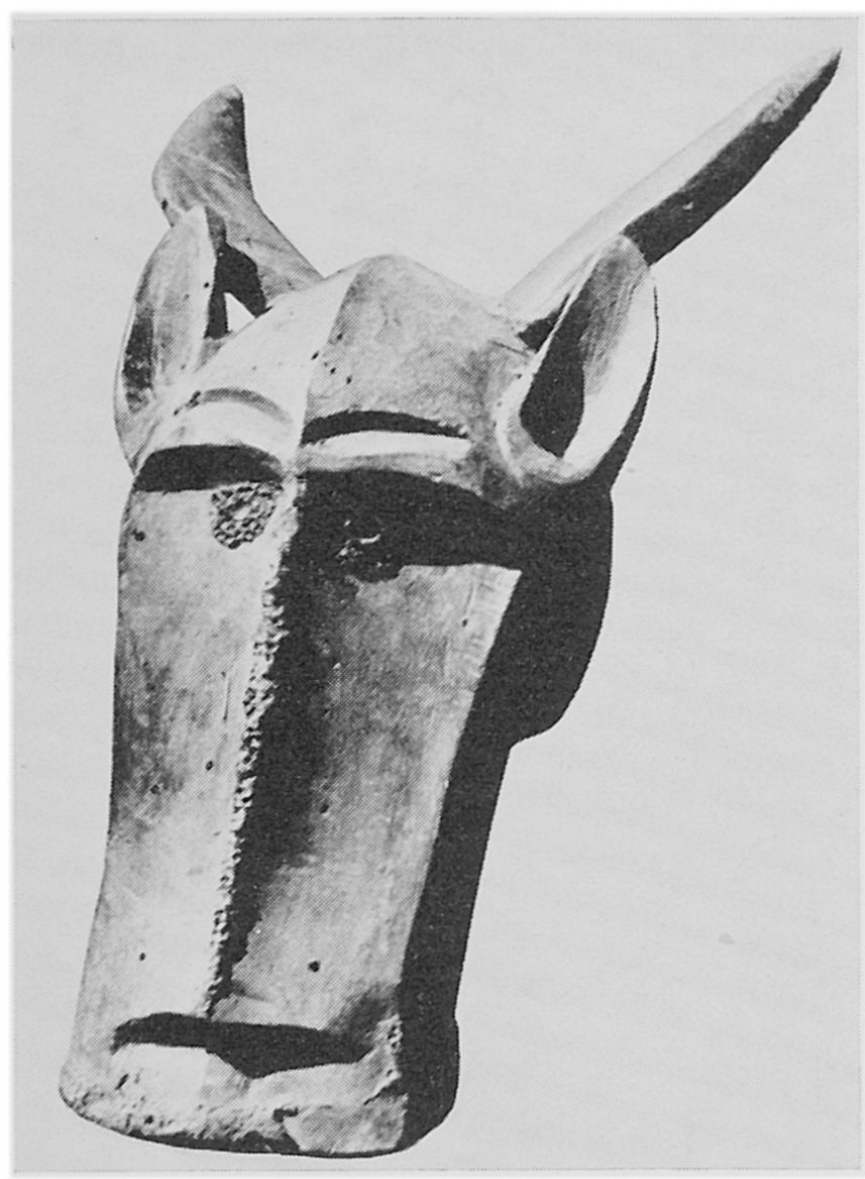

Abb. 6 Buschkuhmaske aus $\mathrm{Holz}$

Stammes -, die Statik, die Symmetrie und der klare, bestimmte Aufbau der als Einzelform wirkenden Körperteile, zudem die Rhythmen, die werten und gliedern. Zusammen bestimmen diese formalen Elemente den Eindruck hoher Ruhe und Feierlichkeit.

Die Proportionen sind nicht nach dem Naturvorbild gemessen, sondern nach dem Bedeutungsgehalt: groß, formal betont sind der Kopf mit dem ernsten Gesicht, die Brust und der Leib mit dem Nabel als Lebenszentrum. Diese Partien sind organisch erfühlt und verraten eine vortreffliche Beobachtungsgabe, beispielsweise der sensible Umriß der Brust. Die Gelenke der Ellbogen und Knie sind an der rhythmischen Gliederung der Figur mitbeteiligt. so daß sie von latenter Bewegung durchpulst erscheint. Die Arme stützen die Schale, die Hände greifen deren Rand wirklich an. Die Beine sind so stämmig und die Füße so wuchtig und undifferenziert gehalten, daß sie ohne gefühlsmäßige Bindung als Sockel wirken, als rein formales Element die Tragfunktion des schweren Körpers glaubhaft ausüben.

Die Oberfläche der Statue ist durch Muster bereichert, um zu schmücken und gewisse Partien auszuzeichnen, wie etwa das sternförmige Bild um den Nabel. Die figürlichen Motive von Eidechse und Chamäleon haben den Sinn, die mythische Lebens- kraft zu erhöhen. Die randständigen Bogen der Schale sind dem Motivschatz der Haussa entlehnt.

Wenn wir die Figur drehen, so erfassen wir weitere wichtige Aspekte der Form: die schöne Rundung des Kopfes mit der Frisur, die edle Nackenlinie der Frau und das heißersehnte Kind. Es sitzt genau an der richtigen Stelle und wirkt als Gegenpol zum schweren Leib der Mutter, ist formal mit ihr verspannt und in ihren Rhythmus eingestimmt. Es wäre falsch, wollte man die Haltung des Kindes, das seinen Kopf nicht wie ein gewöhnlicher Säugling dem Rücken der Mutter anschmiegt, mangelnder Beobachtung zuschreiben; es soll vielmehr seiner Bedeutung entsprechend ein Eigenleben besitzen. Sein Kopf ist aufgerichtet, jedoch klein, hat er doch noch nicht die geistige Bedeutung eines Erwachsenen erlangt. Mit den vergrößerten Händen und Füßen hält sich das Kind kräftig an der Mutter fest. In der Rückenansicht wäre der schöne Bogen zu sehen, den die Schulter- und Kopfpartie des Kindes mit den Armen und der Schale der Frau beschreibt. So ist die Figur nach

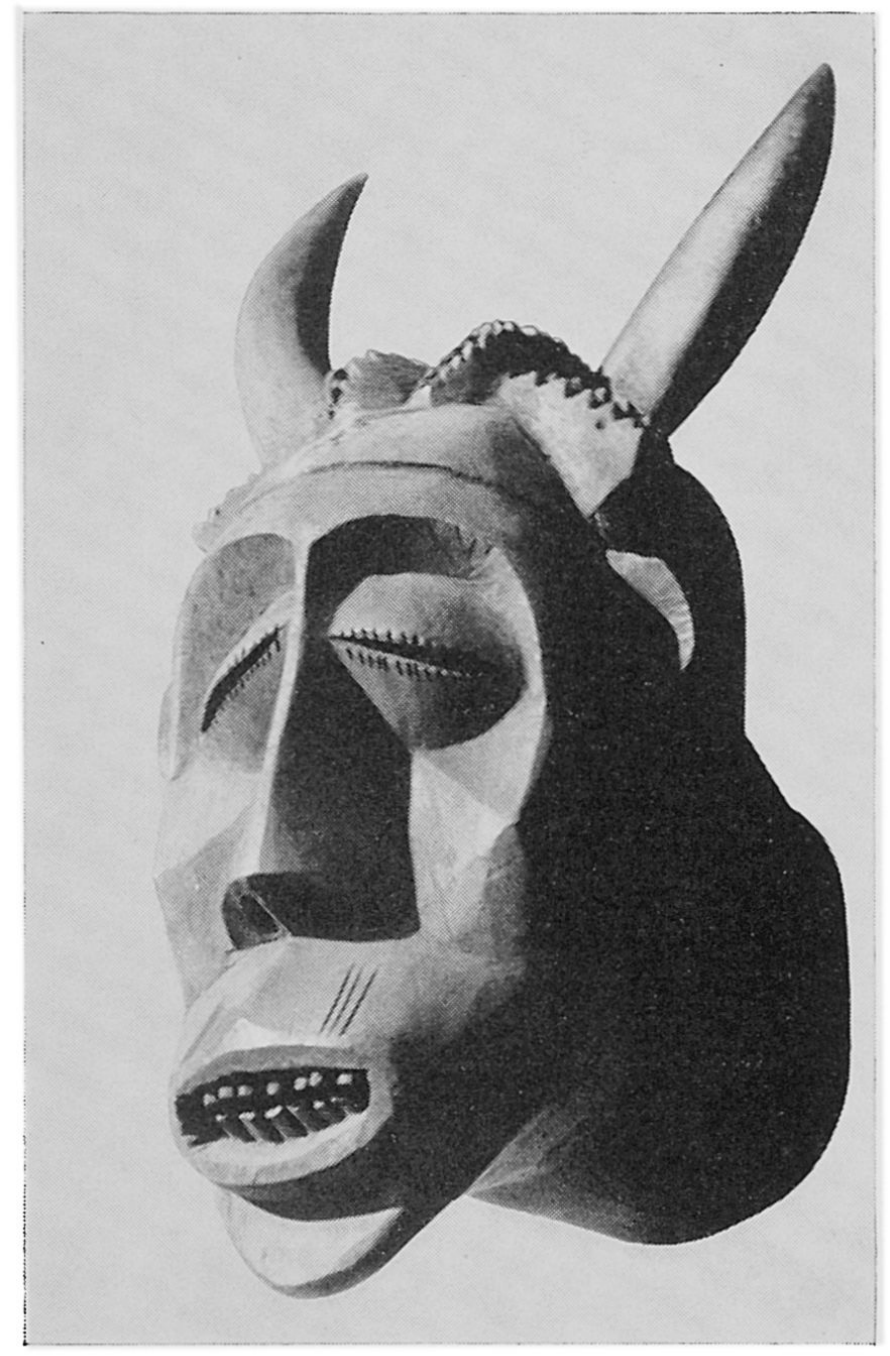

Abb. 7 Buschkuhmaske aus Holz 


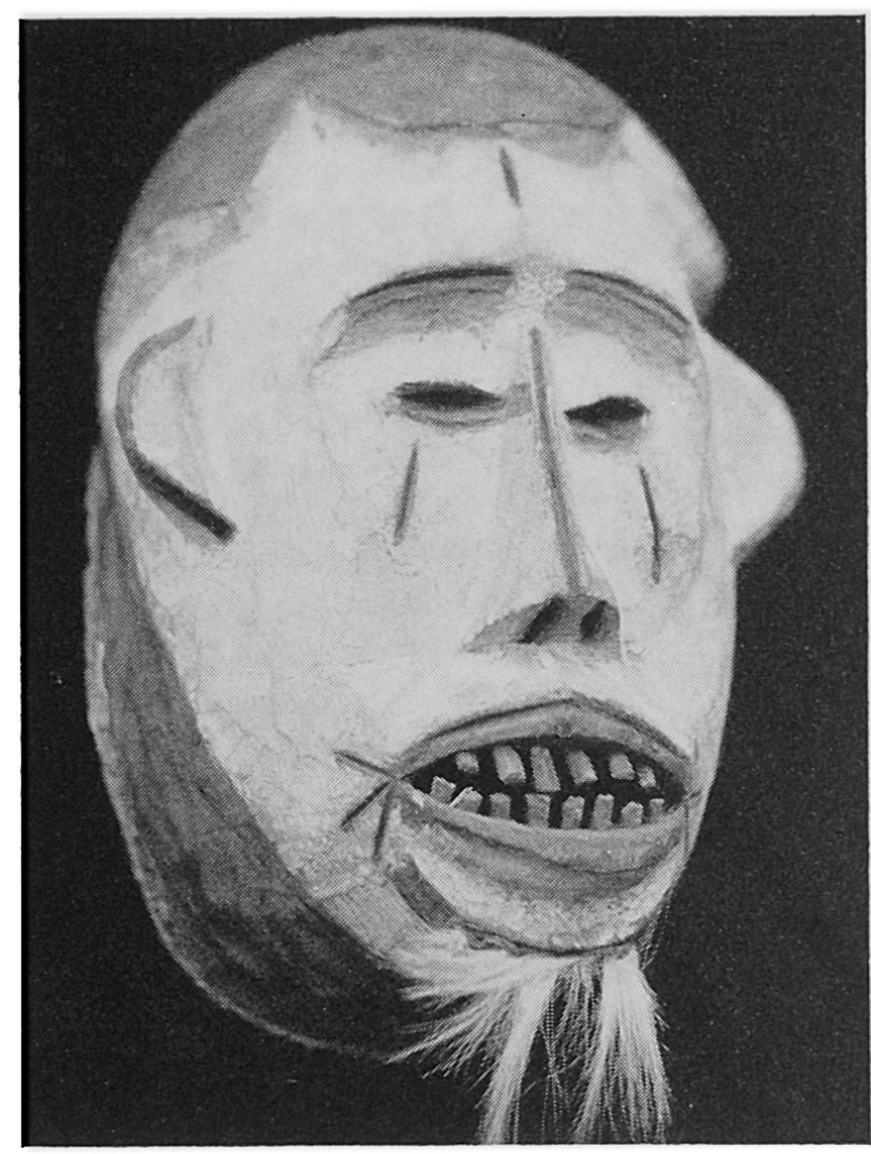

Abb. 8 Weisse Totengeistmaske aus Holz; schwarze Farbakzente und Bart

allen Seiten hin ausgewogen, auch wenn ihre Hauptwirkung auf der Frontansicht beruht.

Besonders schön ist der Kopf der Mutter. Da sind alle Teile in der gleichen, feinen Empfindung aufeinander abgestimmt: die Wölbung der Stirn unter dem Bogen, der sie vom Haar abgrenzt, das organische Ohr, die Wange, die edle Nasenlinie, der scheinbar bebende Mund; und es lebt der Blick zwischen den subtilen Lidern. Jede feine Schwingung der Oberfläche zählt und verleiht dem Werk poetische Stimmung und Harmonie. Die Form ist der überzeugende Ausdruck eines überirdischen Wesens, das lebt. Form und Sinn sind im Einklang. Wir könnten die künstlerische Qualität unserer Mutter-Statue nicht besser demonstrieren als durch den Vergleich mit einer anderen neueren Figur (Abb. 14). Diese ist steif und in allen Teilen flau. Nichts ist durchgestaltet, nichts erlebt. Die Oberfläche ist stumpf, die Augen tote Wülste, der Mund erscheint leblos. Der künstlerische Abfall ist gewaltig.

Das zweite Werk, dem wir unsere Aufmerksamkeit schenken, ist eine besonders originelle Buschkuhmaske (Abb. 7). Sie soll das Talent der AfoKünstler noch von einer andern Seite her beleuchten. Das mythische Wesen, das die Schutzmacht der Gemeinschaft versinnbildlicht, ist mit tierischen und menschlichen Zügen dargestellt. Im Gegensatz zu der poetisch beseelten Mutterstatue liegt die Betonung hier auf dem Ausdruck dynamischer Kraft, auf gesteigerter Expressivität. Diese Wirkung wird durch eine größere Abstraktion erzielt, durch die Verdichtung des Naturvorbildes auf wenige bedeutungsvolle Grundformen.

Die Kraft äußert sich formal besonders durch die starke Licht- und Schattenwirkung der vor- und zurückspringenden, kraftvoll erfaßten Einzelteile, so in der Konkordanz der tiefen Augenhöhlen mit der vorspringenden Mundpartie oder in den aggressiv wirkenden Wangenbeinen. Diese setzen die herben Augenbrauenbogen fort und zielen zur Lippenspitze hin. Diese Lippe fängt auch die vertikale Mittellinie auf. Solche Kurven intensivieren die Gesichtszüge in hohem Maße. Die schräg in der Augenhöhe liegenden plastischen Augen erscheinen lebendig; der Blick wird durch die Strichelung der Lidränder wirksam. Dem geöffneten bezahnten Maul glaubt man, daß es brüllen kann, in tierhaft energiegeladener und trotzdem verhaltener Urkraft. Logik herrscht im Zusammenklang.

Auch die Profilansicht macht die Geschlossenheit und die Einheitlichkeit der herben Durchgestaltung der Formen deutlich. Trotzdem können die Bilder nur einen Teilaspekt der Maske vermitteln: das Lebenselement einer jeden Maske ist ja die Bewegung. Nur im Tanz und mit dem ganzen Maskenaufzug des Tänzers bilden sie ein vollständiges Ganzes. Erst wenn die Lichter der Fackeln in den plastischen Formen dieser Buschkuhmaske spielen,

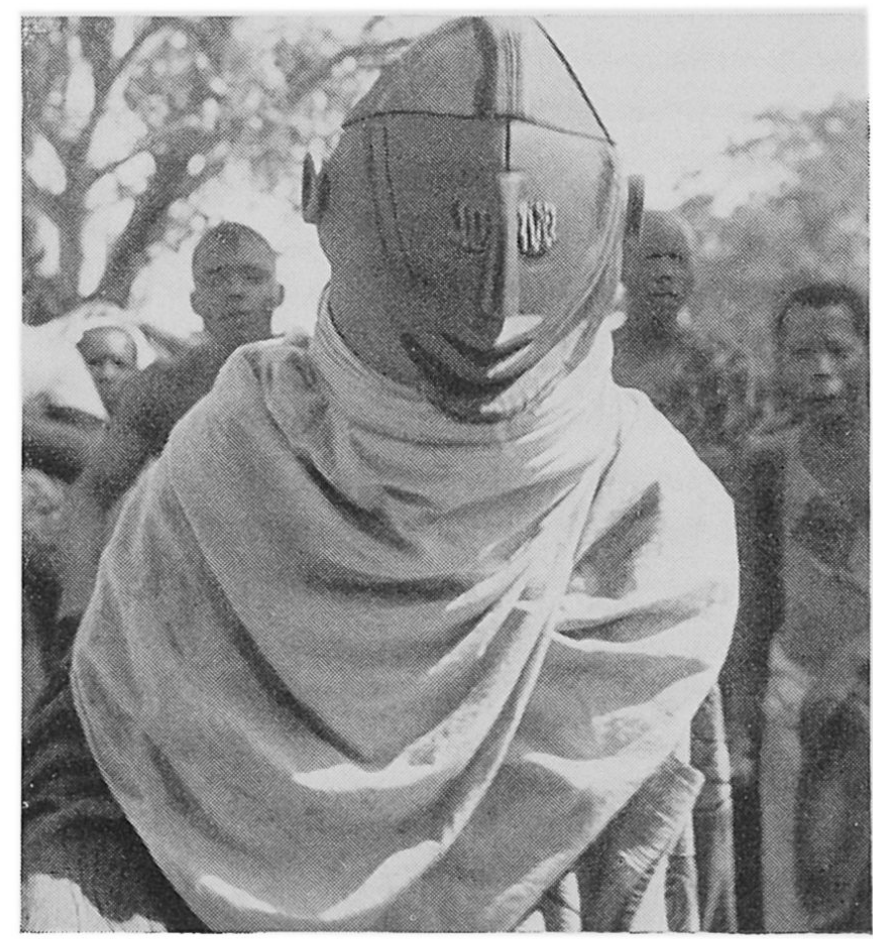

Abb. 9 Tänzer in schwarzer hölzerner Totengeistmaske: Augen aus Abrusbohnen 


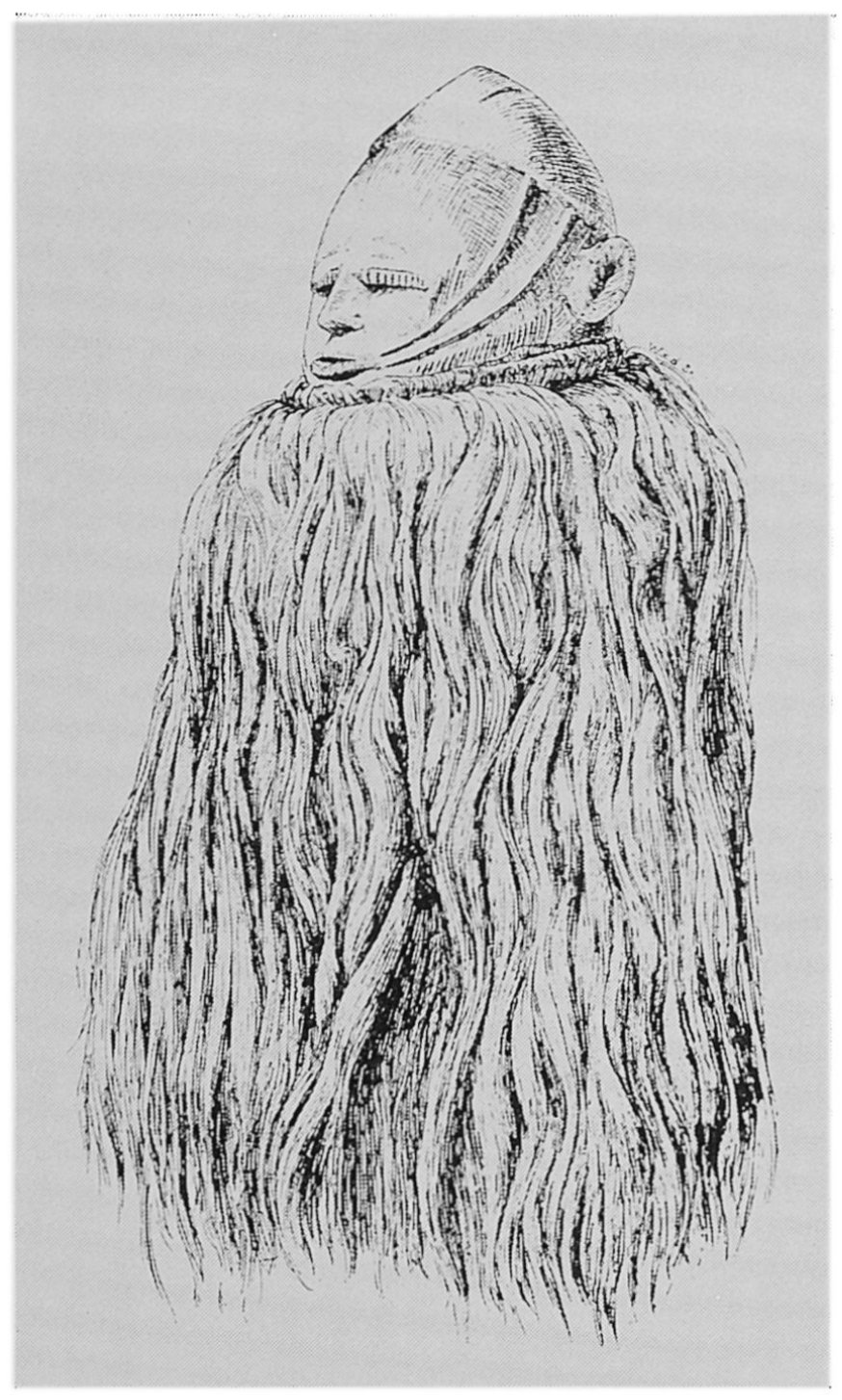

Abb. 10 Zeichnung der Flegelschen Maske aus Wukari (aus Passarge: "Adamaua»)

kommt die Expressivität dieses Geistwesens zur vollen Wirkung.

Angesichts solch ausgewählter Skulpturen glauben wir mit Recht, von einer echten, erlebten Kunst sprechen zu dürfen. Damit ist auch schon die Antwort auf die dritte Frage vorbereitet, auf die Frage nämlich, ob bei einem Stamm, der seine altertünliche Lebensweise so gut bewahrt hat, noch Phasen des Kunstwerdens zu entdecken wären. Die Spitzenwerke der Afo sind zu differenziert, zu gekonnt, um naiv oder roh genannt zu werden; sie ragen weit über all das hinaus, was gemeinhin zur Primärkunst gerechnet wird. Es frägt sich nur, wie die Afo zu ihrem persönlichen Stil gelangt sind; das aber ist weit schwieriger zu erurieren.

\section{Kulturzusammenhang}

Allein schon die Feststellung, daß die Afo-Skulpturen allgemeine Wesenszüge der Negerkunst tra- gen, beweist, daß sie nicht ursprünglich sein können, sondern in einem weiteren Kulturzusammenhang gesehen werden müssen. Und wir brauchen auch nicht lange zu suchen. Nigeria hat ja eine Fülle von Kunststilen hervorgebracht, solche hohen Alters - die Kunst der Yoruba in Südwest-Nigeria läßt sich fast tausend Jahre zurückverfolgen und solche, die an die Gegenwart heranreichen. Unter den Schnitzstilen Süd-Nigerias nun finden wir etliche, die mit unserer Afo-Skulptur stilverwandt erscheinen. Wir denken vor allem an die weißen Masken der Idoma ${ }^{3}$, Ost-Igala und Nord-Ibo, an die bereits erwähnten Ikenga der Igala 4 und Idoma und an einige Schnitzwerke, die aus Wukari, der einstigen Hauptstadt des Jukunreiches in Adamaua südlich des Benue, stammeñ: zwei Statuen des Horniman-Museums in London (Abb. 15), ein von Glauning 1904 gesammelter Figurenthron, der sich im Museum für Völkerkunde in Berlin befindet, und eine Maske, die Flegel im Jahre 1887 nach Berlin mitgebracht hat (Abb. 10). Diese Maske ist den schwarzen Totenmasken der Afo verblüffend ähnlich. Die beiden Figuren des Horniman-Muse-

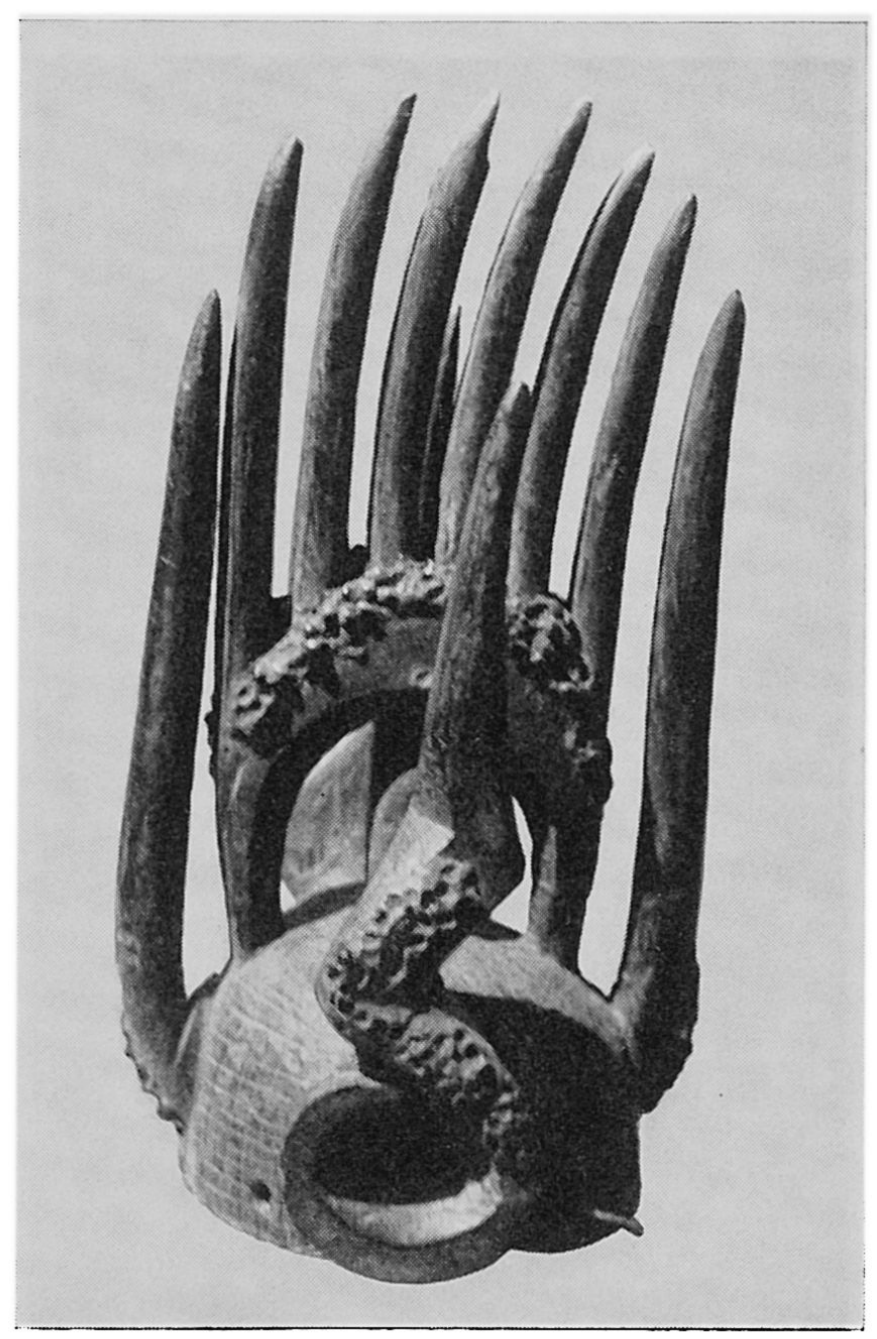

Abb. 11 Tanzaufsatz aus Holz mit Auflage von Abrusbohnen 

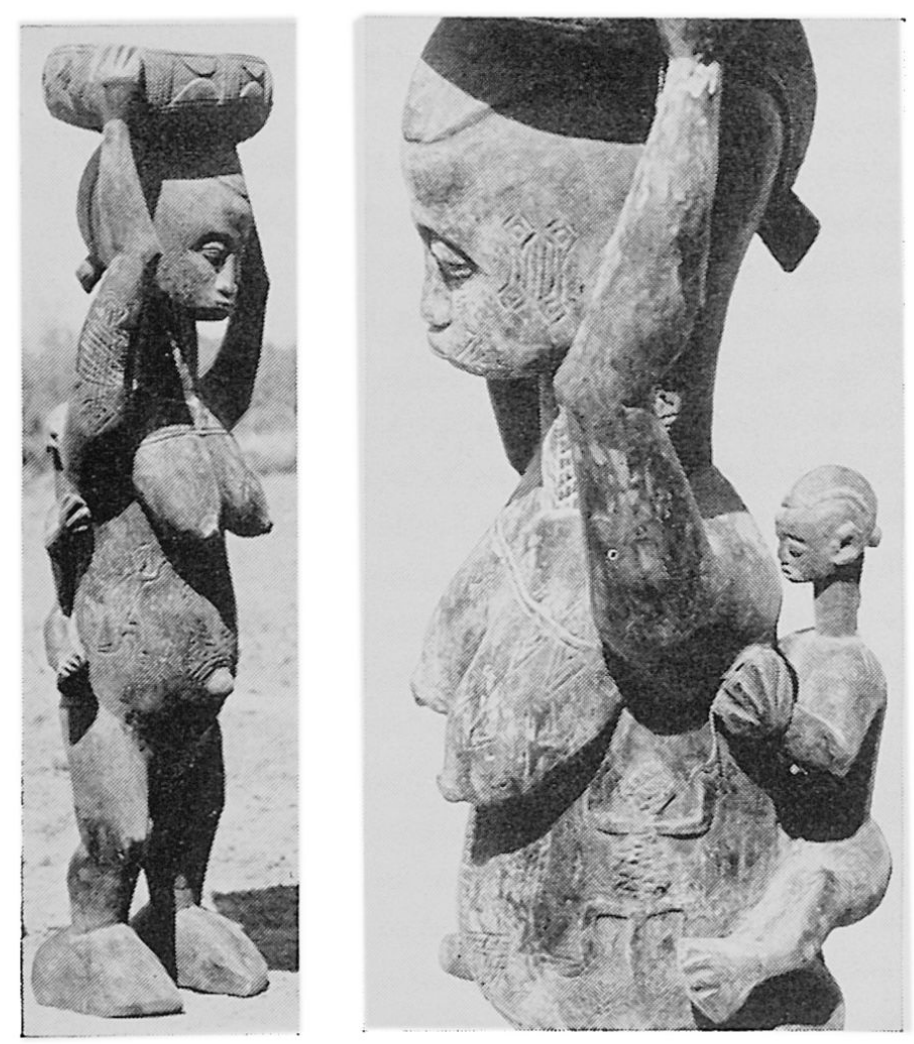

Abb. 12 Fruchtbarkeitsstatue der Afo

Abb. 13 Seitenansicht der selben Statue den Adamaua-Bergen noch im reinen Jägerstadium befunden hätten. Und Jäger pflegen sich ja schwerlich mit großen Skulpturen zu belasten. Es fehlen bei den Afo auch alle Anzeichen der mit dem Jukun-Yoruba-Komplex verbundenen Hochkulturmerkmalen des Gottkönigtums. Andere Elemente wiederum, die die Afo mit den Jukun gemeinsam haben, wie Gräber umschließende Steinkreise, eiserne Gongs, Stelzenläufer, mit Spinnmembran verschlossene Geheiminstrumente zur Nachahmung der Geisterstimme, geschliffene Steinbeile, Büffelkult und Schutzgeisterglauben, sind auch bei andern Stämmen Westafrikas bezeugt.

Angenommen, die Afo hätten sich schon im 19. Jahrhundert als berühmte Schnitzer betätigt: offen bleibt die Frage nach wie vor, wie sie zu ihrer persönlichen Form gekommen sind, zu einer Form, die weder spontan noch unabhängig ist, sondern an alte Traditionen in Nigeria anknüpft. Diesen Zeitpunkt würde ich am ehesten vor hundert Jahren ansetzen, als die Afo im Übergangsstadium vom einfachen Jäger zum Ackerbauer die einfachsten Techniken des Handwerks von ihren höher zivilisierten Nachbarstämmen adaptierten. Da hatten sie wohl auch bereits bestehende Kunstformen aufgegriffen und ihrem Lebensbereich eingegliedert. Bald aber muß sich ihr Schnitztalent durchgesetzt

ums stimmen wohl in manchen Zügen mit den im Nasarawa-Bergland beobachteten Afo-Werken überein, sind jedoch wesentlich dynamischer und eckiger. In ihrer künstlerischen Qualität ragen sie jedoch alle weit über die gewöhnliche Figuralkunst der Benue-Region hinaus und sind diesbezüglich höchstens mit Meisterwerken der Yoruba zu vergleichen. Eine gewisse Yoruba-Affinität der Horniman-Figuren hatte früher einige Forscher bewogen, sie mit Vorbehalten diesen Yoruba zuzuschreiben.

William Fagg aber, der große Kenner nigerianischer Kunst im Britischen Museum, schreibt alle drei Wukari-Skulpturen den Afo zu. Er meint, daß es nicht ausgeschlossen sei, daß die Jukun die Figuren im 19. Jahrhundert bei den als Schnitzer berühmten Afo von Nasarawa bestellten und über eine Distanz von 200 Kilometern hinweg transportieren ließen. Die übrigen Schnitzereien der Jukun sind in einem andern Stil gehalten.

Es würde auf der Hand liegen, die Entstehung der Wukari-Statuen in jenem Zeitraum des 19. Jahrhunderts anzusetzen, als die Afo, unter dem Druck der Fulani-Reiter auf dem südlichen Benue-Ufer Zuflucht suchend, in engeren Kontakt mit den Jukun gerieten. Da hatten sie unter Umständen am Wukari-Hofe für die hamitische oder halbhamitische Jukun-Aristokratie gearbeitet. Es steht jedoch dieser verlockenden Hypothese die Behauptung der Afo gegenüber, wonach sie sich beim Auszug aus
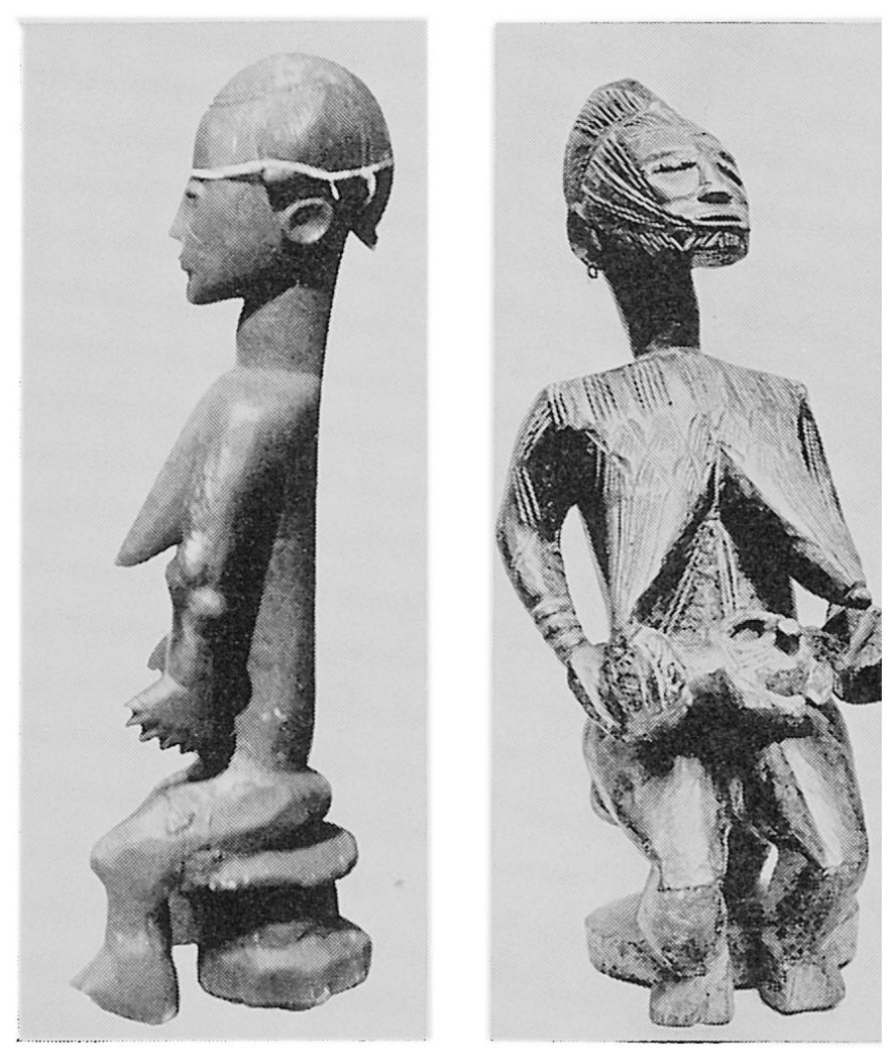

Abb. 14 Neuere Fruchtbarkeitsstatue der Afo Abb. 15 Fruchtbarkeitsstatue aus Wukari, Besitz des Horniman-Museums in London (aus Radin und Sweeney: African Folktales and Sculpture) 
haben, indem sie nicht etwa bei stümperhafter Nachahmung verblieben, sondern die übernommenen Formen mit Energie durchdrangen und in Wechselwirkung von künstlerischem Individuum und Traditionsgebundenheit ihren hervorragenden persönlichen Stil prägten, der in weitem Umkreis Geltung errang.

Die Kulturgeschichte Afrikas ist vielschichtig, reicht Jahrtausende zurück. Den Anfängen seiner Kunst nachzuspüren, ist nicht mehr möglich, dafür ist es in Afrika zu spät; allzu viel ist für immer verloren. Das Kunstschaffen ist zudem seit langem von Hochkulturelementen befruchtet worden und deshalb weit davon entfernt, primitiv oder gar ursprünglich zu sein. Die wirklichen Primitivvölker in den afrikanischen Rückzugsgebieten haben keine figurale Kunst.

\section{Zusammenfassung}

So kommen wir aufgrund der bei den Afo gemachten Beobachtungen zum Ergebnis, daß wir lediglich das Endstadium der traditionellen Negerkunst erfassen können, selbst bei einem isolierten Bergstamm, der seine altbewährten Glaubensformen noch bewahrt hat, wie im Falle der Afo. Dieser Stamm, der in seinem Jägerstadium kaum nennenswerte Kunst übte, hat beim Ưbergang zum Ackerbau von Völkern höherer Kultur fertige Kunstformen übernommen. Die Afo waren aber begabt genug, um diese Fremdformen sinnvoll in ihr eigenes Leben und in ihre Vorstellungswelt einzubauen, durch Erlebniskraft zu spannen und in der Isolation des Busches zu einem persönlichen Stil von künstlerischem Rang zu gelangen. Eine ungefüge, undifferenzierte Primärkunst läuft unbekümmert nebenher.

\section{Anmerkungen}

1 Es gereicht mir zur besonderen Ehre, zum 75. Geburtstag meines verehrten Lehrers und verständnisvollen Förderers Prof. Dr. Steinmann Teilergebnisse meiner Feldforschung in Nord-Nigeria vorlegen zu dürfen. Die Expedition, die Jolantha Neukom-Tschudi und ich 1954/55 unternahmen, hatte den Zweck, einen noch unerforschten Negerstamm ethnologisch zu bearbeiten und für unsere Museen zu sammeln. Die Wahl fiel auf die Afo, weil einige ihnen zugeschriebene Skulpturen die Hoffnung nährten, Neuland der Kunst entdecken zu können.

2 Fagg 1965: Abb. 40.

3 Sieber: Abb. 22; Fagg 1963: Abb. 136.

4 Fagg 1965: Abb. 40.

5 Elisofon-Fagg: Abb. 149: Fagg 1963: Abb. 142, 143 a, b; Fagg 1965: Abb. 42; Radin: Abb. 60; Trowell: Abb. 20.

\section{Einige Literaturhinweise}

Elisofon, E. und Fagg, W.: Die afrikanische Plastik. Köln 1958. - Fagg, W.: Bildwerke aus Nigeria. München 1963; Afrika, 100 Stämme - 100 Meisterwerke (Ausstellungskatalog) Berlin 1964; Tribes and forms in african art. Paris 1965. Goldwater, R.: Bambara. New York 1960. Meek, C. K.: A Sudanese Kingdom. London 1931. - Passarge, S.: Adamaua. Berlin 1895. - Radin, P. und Sweeney, D. J.: African Folktales and Sculpture. New York 1952. — Sieber, R.: Sculpture of Northern Nigeria. (Katalog des Museum of Primitive Art) New York 1962. - Steinmann, A.: Das Chamäleon in der Kunst Westafrikas. In: Atlantis, 1951, Heft 10. - Trowell, M.: Classical African Sculptures. London 1964. - Tschudi, J.: Aus dem sozialen Leben der Afo, Hügelland von Nasarawa, Nigeria. In: Baessler-Archiv, Neue Folge, Band IV. 